\title{
Validation of "Care of the Dying Evaluation" in Emergency Medicine (CODE-EM): pilot phase of end-of-life management protocol offered within emergency room (EMPOWER) study
}

\author{
Mui Teng Chua ${ }^{1,2} \wedge$, Win Sen Kuan ${ }^{1,2} \wedge$, Charles Qishi Zheng ${ }^{3}$, Ling Tiah ${ }^{4}$, Ranjeev Kumar ${ }^{5}$, \\ Yoko Kin Yoke Wong ${ }^{3}$, Jingping Lin ${ }^{1}$, Sufang Liang ${ }^{1}$, Catriona R. Mayland ${ }^{6,7}$, Luming Shi ${ }^{3,8}$, \\ Irwani Ibrahim $^{1,2}$, Rakhee Yash Pal ${ }^{1,2}$
}

${ }^{1}$ Emergency Medicine Department, National University Hospital, National University Health System, Singapore, Singapore; ${ }^{2}$ Department of Surgery, Yong Loo Lin School of Medicine, National University of Singapore, Singapore, Singapore; ${ }^{3}$ Singapore Clinical Research Institute, Singapore, Singapore; ${ }^{4}$ Accident \& Emergency Medicine, Changi General Hospital, Singapore, Singapore; ${ }^{5}$ Acute and Emergency Care Centre, Khoo Teck Puat Hospital, Singapore, Singapore; ${ }^{6}$ Department of Oncology and Metabolism, University of Sheffield, Sheffield, UK; ${ }^{7}$ Palliative Care Institute, University of Liverpool, Liverpool, UK; ${ }^{8}$ Centre for Quantitative Medicine, Duke-NUS Medical School, Singapore, Singapore Contributions: (I) Conception and design: MT Chua, WS Kuan, L Tiah, CR Mayland, I Ibrahim, R Yash Pal; (II) Administrative support: MT Chua, WS Kuan, L Tiah, R Kumar, J Lin, S Liang, I Ibrahim, R Yash Pal; (III) Provision of study materials or patients: L Tiah, R Kumar, R Yash Pal; (IV) Collection and assembly of data: L Tiah, R Kumar, J Lin, R Yash Pal; (V) Data analysis and interpretation: CQ Zheng, YKY Wong, L Shi; (VI) Manuscript writing: All authors; (VII) Final approval of manuscript: All authors.

Correspondence to: Dr. Mui Teng Chua. Consultant, NUH Emergency Medicine Department, 9 Lower Kent Ridge Road, Level 4, Emergency Medicine Department Office, Singapore 119085, Singapore. Email: mui_teng_chua@nuhs.edu.sg.

Backgrounda An increasing number of patients who present to emergency departments are at their end-
of-life phase and have significant palliative care needs such as in symptom control for pain and dyspnoea.
Evaluating quality of care provided is imperative, yet there is no suitable tool validated in the emergency
and Asian settings. We aim to examine the face and construct validity, and reliability of a newly developed
questionnaire, Care of the Dying Evaluation - Emergency Medicine, for measuring the quality of end-of-life
care in an Asian emergency context.

Methods: A mixed methods pilot study was conducted. Participants composed of the next-of-kin to thirty dying patients who presented to the emergency departments of three public hospitals in Singapore. Qualitative evaluation, using cognitive "think-aloud" interviews, and quantitative analysis were employed. Percentage agreement and $\kappa$ statistic were measured to evaluate temporal stability of the questionnaire. Cronbach's $\alpha$ and item-total correlations were used to assess internal consistency within the constructs. Confirmatory factor analysis was performed for construct validity.

Results: All participants reported clear understanding of the questionnaire with no ambiguity; a minority felt the questions caused emotional distress (7/30, 23.3\%). The questions showed moderate to good testretest reliability. Internal consistencies within the constructs were good for "ENVIRONMENT" and "CARE", and moderate for "COMMUNICATION". Factor loadings range from 0.40 to 0.99 .

Conclusions: The Care of the Dying Evaluation - Emergency Medicine questionnaire may be valid and reliable for use in an Asian emergency setting. Our prospective multicentre study using this evaluation tool may provide more insight on the quality of care rendered to dying patients and identify areas for improvement.

Trial registration: ClinicalTrials.gov (NCT03906747).

Keywords: Palliative care; terminal care; hospital emergency services; quality of care

^ ORCID: Mui Teng Chua, 0000-0002-6326-4914; Win Sen Kuan, 0000-0002-2134-7842. 
Submitted Feb 13, 2021. Accepted for publication Apr 28, 2021.

doi: 10.21037/apm-21-380

View this article at: http://dx.doi.org/10.21037/apm-21-380

\section{Introduction}

Globally, the population is ageing, with the number of persons aged 80 years and above projected to rise to 425 million by 2050, a three-fold increase from 2017 (1). Consequently, an increase in chronic illnesses and comorbidities is prevalent among patients presenting to the emergency departments (EDs), rendering the care of such patients to be more complex. More patients will be attending EDs for symptom control, mental distress, ease of access to healthcare and caregiver stress at their end-of-life phase $(2,3)$, which is defined by the European Society for Emergency Medicine as patients facing a rapid deterioration in health with imminent death in an emergency medicine setting (4). Such critically ill and dying patients have significant palliative care needs that include management of moderate to severe symptoms of pain, fatigue and dyspnoea (3). Apart from infrastructural constraints due to its inherent chaotic and overcrowded environment $(5,6)$, emergency physicians are also inadequately trained in pain and symptom management for such patients (7).

While some efforts have been undertaken to establish protocolised management pathways for ED end-of-life patients, quality of care is still not optimised and more can be done (8). To cope with changing demands in healthcare needs in the EDs, the assessment of quality of care rendered to end-of-life patients is particularly important to identify areas for improvement to ensure a good death. One such available instrument is the "Care of the Dying Evaluation" $\left(\mathrm{CODE}^{\mathrm{TM}}\right)$, a shortened and validated version of "Evaluating Care and Health Outcomes - for the Dying" which measures components relating to best practice for care of the dying, previously validated in a Caucasian population within the community settings (9).

$\mathrm{CODE}^{\mathrm{TM}}$ is a 40 -item self-administered questionnaire that evaluates the quality of care in the last days of life and immediate post-bereavement period. Within $\mathrm{CODE}^{\mathrm{TM}}$, three constructs, 'CARE', 'ENVIRONMENT' and 'COMMUNICATION', are examined in detail. However, it has not been validated in a predominantly Asian population and was not administered in an ED setting. Differences in perspectives and attitudes towards end-of-life care are known to exist among various ethnic groups $(10,11)$, and these differences may be more apparent among Asians who are generally thought to be more conservative and reserved in exploring end-of-life issues due to cultural and religious beliefs $(12,13)$. Furthermore, the experience and interaction of patients and family members with the clinical team in ED may contrast with their regular palliative or hospice care providers as there is no pre-existing patient-physician relationship, and ED physicians are less adept at dealing with death-related issues (14). We aim to validate the use of the $\mathrm{CODE}^{\mathrm{TM}}$ questionnaire in the EDs of a multi-ethnic Asian population in Singapore.

This study constitutes the pilot phase of our multicentre study, "End-of-life Management Protocol Offered Within Emergency Room" (EMPOWER); the final and complete study protocol has been published separately (15). The objectives of this pilot were to examine the face and construct validity, and reliability of a newly developed questionnaire for measuring the quality of end-of-life care in EDs in the Asian context, taking reference from the CODE $^{\mathrm{TM}}$ questionnaire (9).

We present the following article in accordance with the STROBE reporting checklist (available at http://dx.doi. org/10.21037/apm-21-380).

\section{Methods}

\section{Study design}

We conducted a mixed methods study between January and April 2019 at the EDs of three public hospitals [National University Hospital (NUH), Changi General Hospital (CGH) and Khoo Teck Puat Hospital (KTPH)] in Singapore. The study was conducted in accordance with the Declaration of Helsinki (as revised in 2013). Ethics approval was obtained from the National Healthcare Group Domain Specific Review Board (DSRB reference no: 2018/00838) and the study protocol was registered with ClinicalTrials. gov (NCT03906747). All enrolled participants provided written informed consent.

\section{Study setting}

The public hospitals included in this study, namely NUH, 
CGH and KTPH, belong to the three main healthcare clusters in Singapore - the National University Health System, Singapore Health Services and National Healthcare Group, which serve the country's western, eastern and northern populations, respectively (16). Each of these three hospitals are tertiary centres with annual ED census of more than 100,000 attendances.

\section{Patient selection}

Next-of-kin of patients who fulfilled all the following inclusion criteria were invited to participate:

* Actively dying patient or high likelihood of mortality within the current admission (based on attending physician's clinical judgement using available clinical data);

* Family accepts that the goals of care are provision of comfort, symptom relief and respect of dignity;

* Patient is not a candidate for cardiopulmonary resuscitation, endotracheal intubation or transfer to the intensive care unit due to medical futility from acute or underlying medical conditions (these include patients who may already have do-not-resuscitate orders established before coming to ED or after thorough assessment upon arrival to ED);

* Any of the life-limiting conditions: chronic frailty with poor functional state and limited reversibility [Karnofsky Performance Scale (KPS) <40\%] (17); chronic severe illness with poor prognosis [terminal cancer, end-stage renal failure (refusal or withdrawal of dialysis), end-stage respiratory, heart or liver disease, advanced neurological disease]; or, acute severe catastrophic conditions and at risk of dying with complications that are not reversible, as subject to the treating clinician's judgement.

We excluded the following subjects: vulnerable population (for example prisoners and pregnant women); refusal to participate; patients who have been recruited, or had declined participation during the previous ED attendance(s); patients in peri-arrest state; and/or family members who are not present at the patient's bedside.

\section{Study procedure}

Participants, i.e., next-of-kin of end-of-life patients, were requested to complete the newly developed questionnaire renamed "Care of the Dying Evaluation - Emergency
Medicine" (CODE-EM) (Appendix 1), derived using the original 40-item $\mathrm{CODE}^{\mathrm{TM}}$. The questions were selected due to their relevance to the ED settings and the other items were removed as they were not applicable in our area of practice. Wordings of the original questions were also rephrased as required to fit the ED context. Details of which questions were omitted or amended and the rationale for doing so are illustrated in Table S1. This first questionnaire completion was done at bedside in the EDs after the patients had received treatment, before or shortly after transfer to wards, terminal discharge from the EDs (where patients passed away at home) or death occurring in EDs.

After completion of the questionnaire, an interview about their experience was conducted by trained research assistants to prompt participants to articulate their thoughts (the "think-aloud" method for cognitive interviews) as they read and answered the questions (18). This helped to improve our knowledge about whether the questions had been understood and how answers had been formulated, in terms of language, length, timing and relevance. Additionally, a standard set of interview questions was asked as a combined approach to elicit its clarity and appropriateness. The key questions included in the interview are as follows:

(I) Were the questions easy to understand and was the wording clear?

(II) Did the questions make you feel emotionally distressed?

(III) Were any of the questions irrelevant?

(IV) What were your thoughts on the length of this survey?

(V) Was the survey conducted at an appropriate timing?

(VI) Any other feedback you would like to share?

For those who were willing to complete the questionnaire for a second time, the second interview was conducted by phone or by mail with a return envelope one month later.

\section{Data collection}

The questionnaires and interviews were conducted by trained research assistants at each study site and responses recorded real-time on standardized paper-based case report forms. Data collected is then entered anonymously into an electronic database in the Research Electronic Data Capture (REDCap) system and maintained at the Singapore Clinical Research Institute's secured server. 


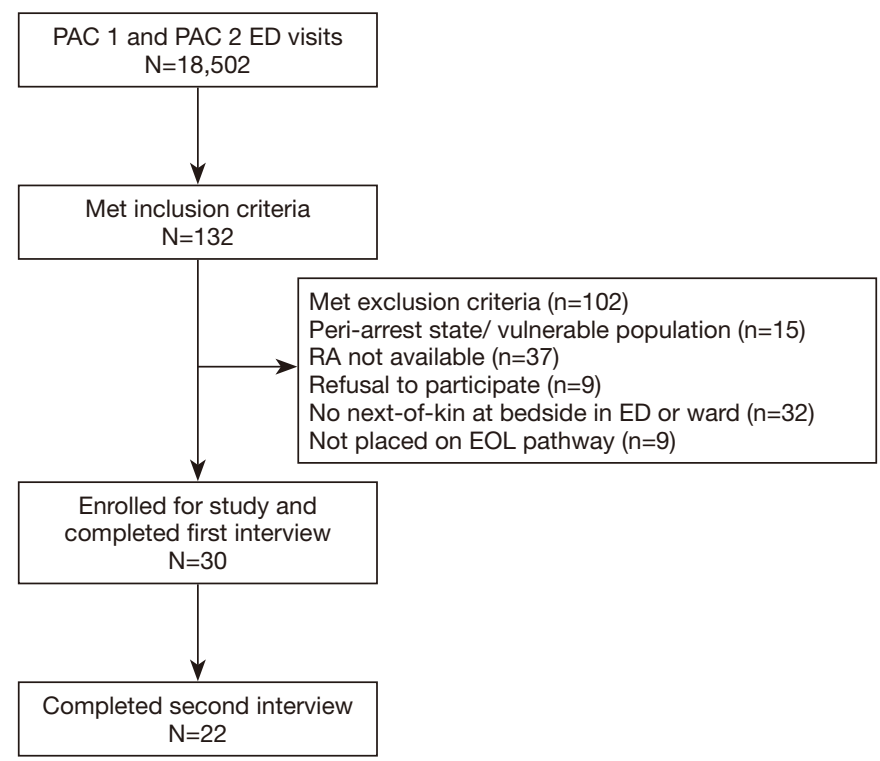

ED - emergency department; EOL - end-of-life; PAC - patient acuity category; RA - research assistant; PAC 1 were patients with imminent cardiorespiratory compromise, requiring immediate attention while PAC 2 were those who require urgent attention, failing which deterioration is likely.

Figure 1 Flowchart illustrating patient screening and enrolment.

\section{Statistical analysis}

The interviews about the experience of completing the questionnaire was recorded. To ensure data integrity, a random selection of completed questionnaires and written interview transcripts $(\mathrm{n}=15)$ were independently reviewed to check for data entry errors by a study investigator (MTC) not directly involved in data collection; any discrepancy was verified and discussed with a third independent investigator (WSK).

Quantitative analysis was carried out using $R$, version 4.0.0 (R Foundation for Statistical Computing, Vienna, Austria). The temporal stability of the developed questionnaire, CODE-EM, was assessed using the following measures: percentage agreement and $\kappa$ statistic (Cohen's for nominal response options and weighted for ordinal response options). As the kappa might not be reliable for rare observations, the criteria for good stability over time are defined as percentage agreement $>70 \%$ or $\kappa>0.60$ and moderate stability over time as percentage agreement $>30 \%$ or $\kappa>0.40(19,20)$. Cronbach's $\alpha$ and item-total correlations were measured to assess internal consistency within the three constructs of "CARE", "ENVIRONMENT" and "COMMUNICATION". Confirmatory factor analysis was used to assess construct validity. The suitability of questions was examined by inspection of the Comparative Fit Index
(CFI).

\section{Results}

\section{Participants' and patients' characteristics}

During the enrolment period, there were 18,502 eligible patient visits and 132 patients fulfilled our inclusion criteria; 102 patients were excluded due to various reasons (Figure 1). A total of 30 bereaved next-of-kin (participants) agreed to participate $(76.9 \%)$. All of them completed the CODE-EM questionnaire and were interviewed in the first assessment; 22 of them (73.3\%) completed the CODE-EM questionnaire a second time one month later (Table 1). Just over half of the end-of-life patients $(17 / 30,56.7 \%)$ were male while the participants comprised more females (16/30, $53.3 \%)$. There was a predominance of Chinese ethnicity among both patients and participants (Table 1). A summary of their baseline demographics is illustrated in Table 1. Most of the deceased patients had chronic frailty as the predominant death trajectory $(19 / 30,63.3 \%)$, followed by sudden death $(5 / 30,16.7 \%)$, cancer $(4 / 30,13.3 \%)$ and organ failure $(2 / 30$, $16.7 \%)$. Patients experienced multiple symptoms, with dyspnoea affecting two-thirds $(20 / 30,66.7 \%)$, while others experienced drowsiness $(16 / 30,53.3 \%)$, weakness or fatigue $(11 / 30,36.7 \%)$, excessive secretions $(7 / 30,23.3 \%)$, terminal 
Table 1 Summary of end-of-life patients and participants' characteristics and interview results

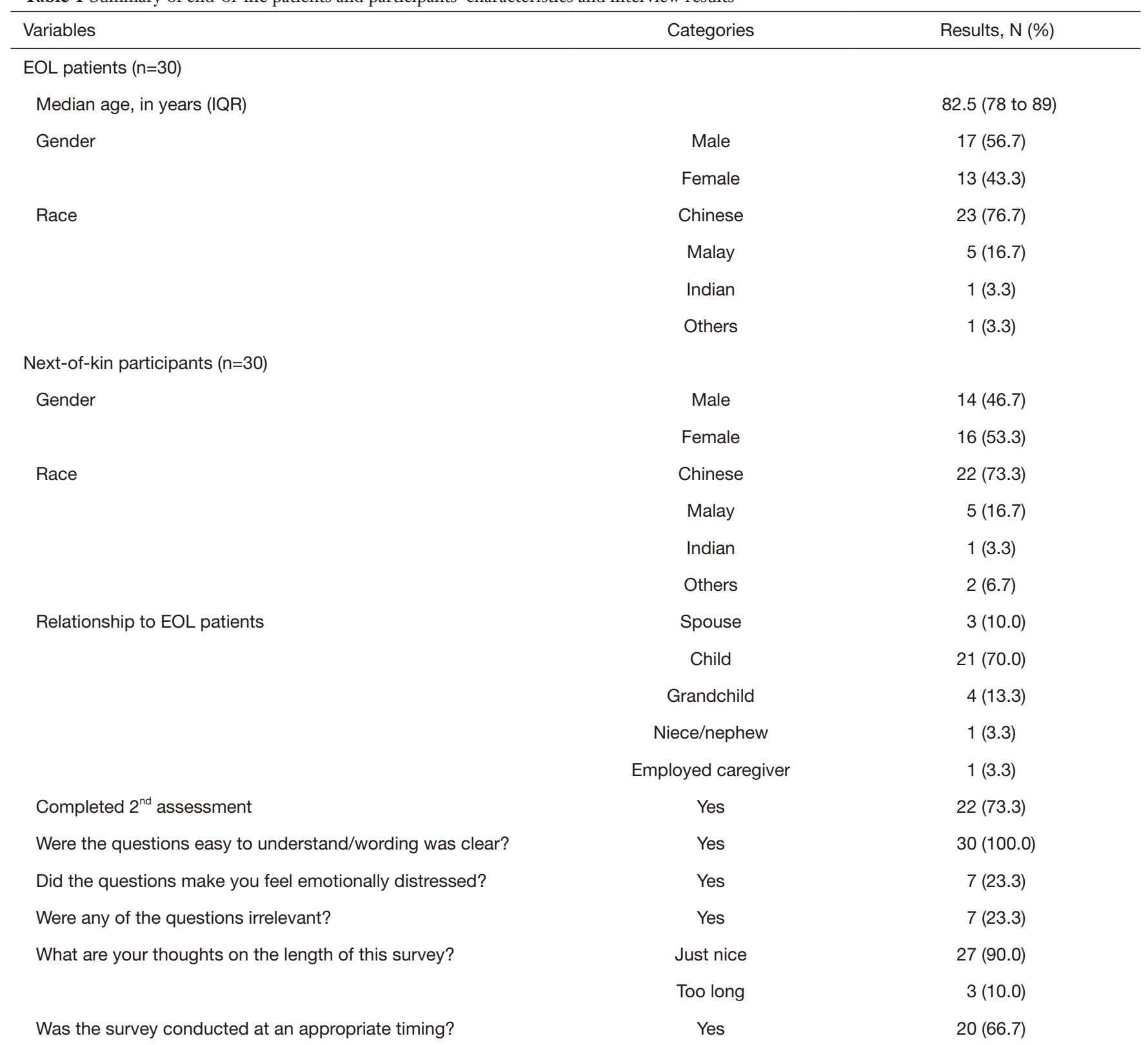

EOL, end-of-life; IQR, interquartile range. Results presented in $\mathrm{n}(\%)$ unless otherwise stated. EOL patients are actively dying patients or patients who have high likelihood of mortality within the current admission. Next-of-kin participants refer to the next-of-kin of these EOL patients; next-of-kin participants completed the Care of the Dying Evaluation - Emergency Medicine (CODE-EM) questionnaire.

restlessness (5/30, 16.7\%), delirium $(5 / 30,16.7 \%)$, cough $(4 / 30,13.3 \%)$ and vomiting $(2 / 30,6.7 \%)$.

\section{Interview results}

All the participants reported a clear and easy understanding of the questionnaire with unambiguous wording. Only a minority felt that the questions made them emotionally distressed (7/30, 23.3\%) (Table 1); among them, some generally felt disturbed as the questionnaire involves discussion of death and particularly in Q19 (which asks if the next-of-kin was informed that the patient would die soon) where a strongly emotive word, "die," was used.

Seven participants $(23.3 \%)$ perceived that some of the 
Table 2 Results of rest-retest reliability

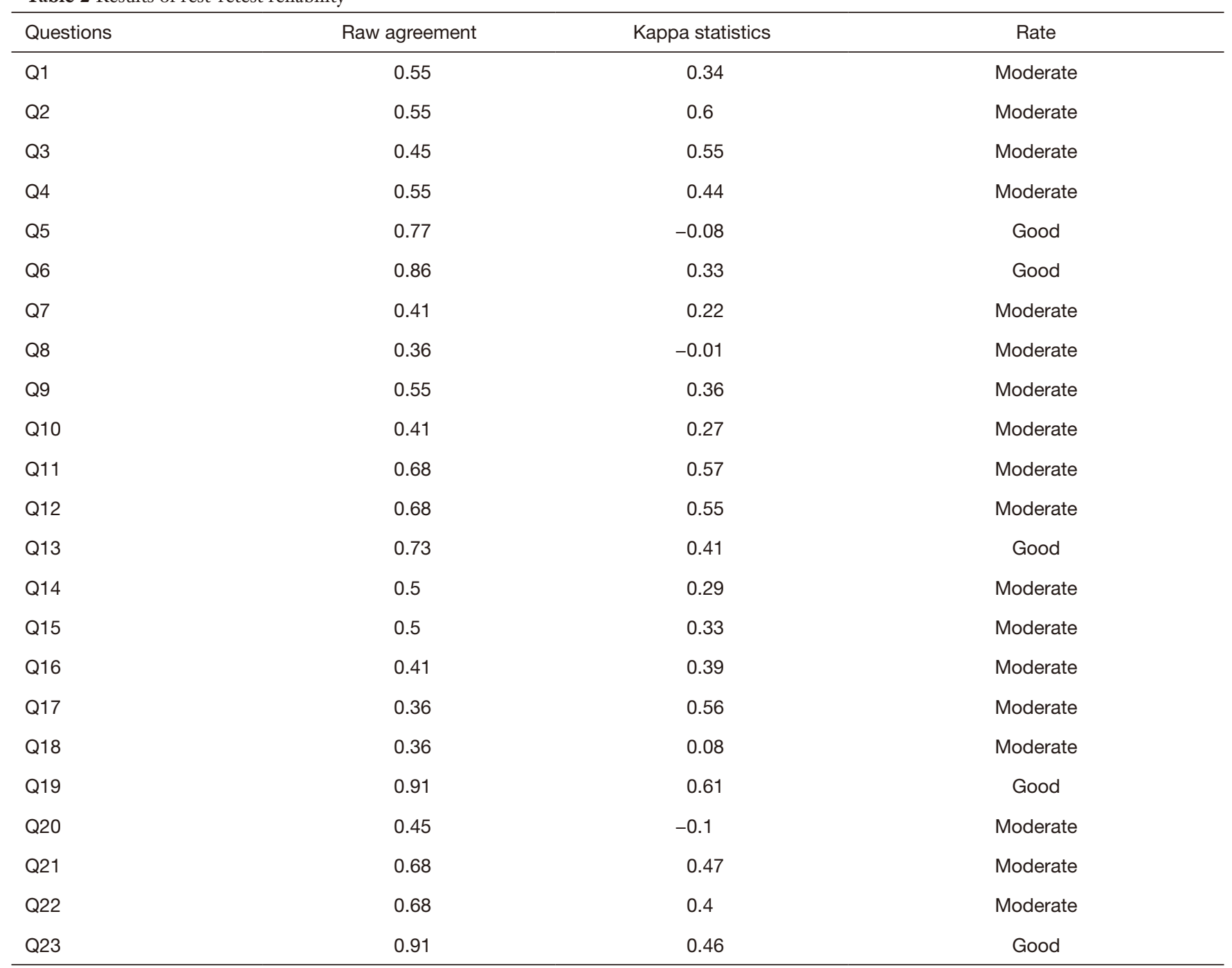

Tables S1 and S2 illustrate the questions in CODE-EM and the modifications from original CODE ${ }^{\mathrm{TM}}$, respectively.

questions were irrelevant. One example was a participant who considered Q7 (which enquires if the patient appears to be in pain) extraneous as he was unable to tell if the unconscious patient was in pain and suggested that the study team tailor the questions to cater for such circumstances.

Many of the participants $(27 / 30,90.0 \%)$ thought the length of the survey was "just nice", while 3 of them felt it was "too long". Two-thirds of the participants (20/30, $66.7 \%$ ) reported that the survey was conducted at an appropriate timing. For those who responded that the survey should be conducted later, there was no consensus on the best possible timing. More details on the interview answers with open questions are summarised in Table S2.

\section{Test-retest reliability}

Two statistics measuring test-retest reliability, i.e., raw agreement and kappa statistics, are reported in Table 2. Negative kappa values were obtained for Questions 5, 8 and 20, which indicate that kappa did not function well in these questions and we had to rely solely on raw agreement. Questions that explored the participants' trust and confidence in ED nurses and doctors (Q5 and Q6), whether patients appeared to have breathing difficulty (Q13), communication regarding imminent death (Q19) and overall support given in ED (Q23) showed "good" testretest reliability. All other questions achieved "moderate" reliability. 
Table 3 Result of internal consistency and construct validity

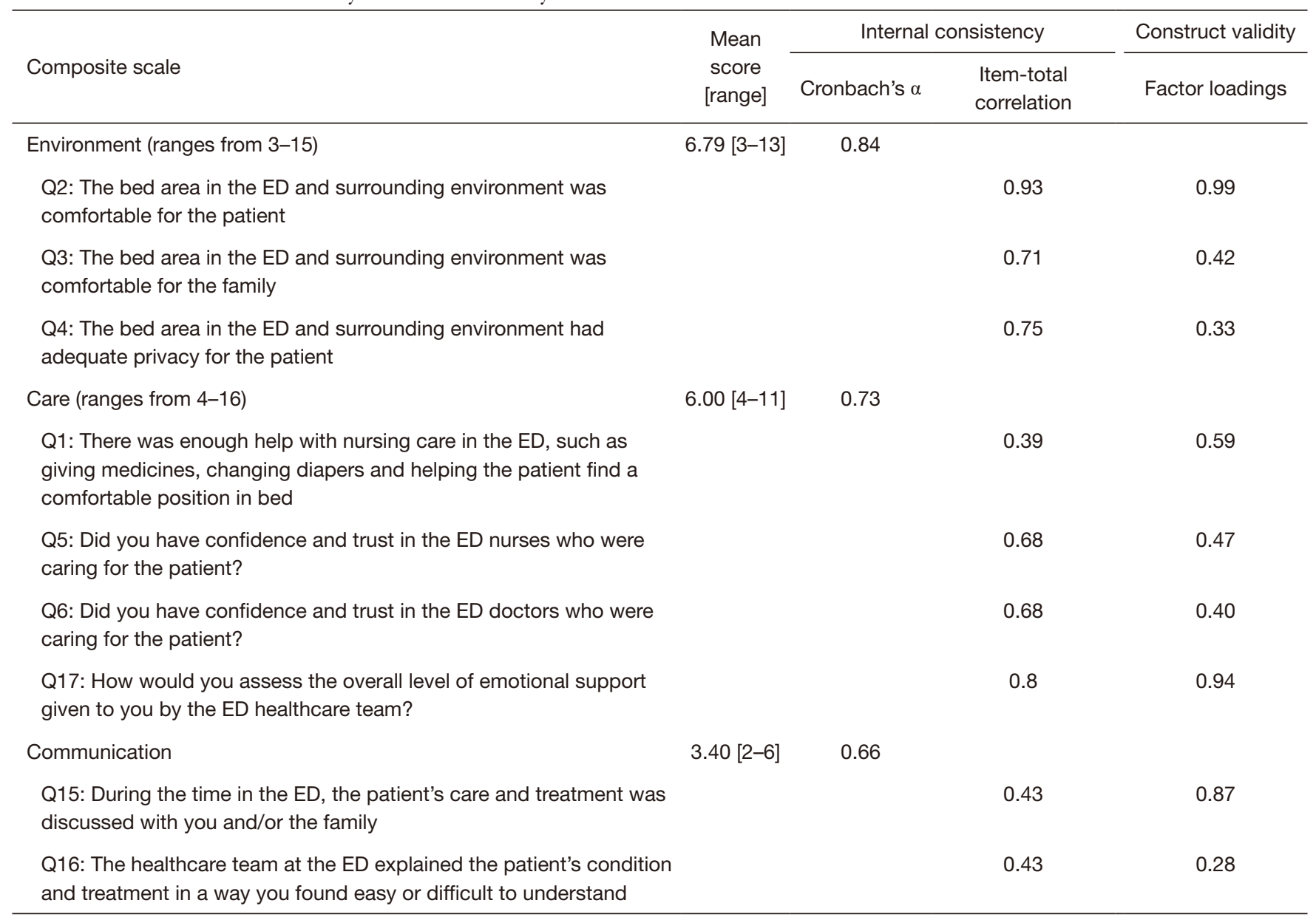

ED, emergency department.

\section{Internal consistency}

The internal consistency was good for "ENVIRONMENT" (Cronbach's $\alpha=0.84$ ) and "CARE" (Cronbach's $\alpha=0.73$ ) suggesting that the inter-item correlations were high, and the items were reliable as individual scales (Table 3). However, the internal consistency of "COMMUNICATION" was moderate with a Cronbach's $\alpha$ of 0.66 .

\section{Construct validity}

The Comparative Fit Index (CFI) was 0.87 confirming suitability of the data for factor analysis. The factor loadings for most of the questions were relatively high, ranging from 0.40 to 0.99 , except Question 16 (factor loading $=0.28$ ) and Question 4 (factor loading $=0.33$ ) (Table 3).

\section{Discussion}

The results from this pilot study support the feasibility of the use of $\mathrm{CODE}^{\mathrm{TM}}$ in a culture (multi-racial Asian population) and environment (ED setting) that is vastly different from its original validation cohort (9). In our sample, CODE-EM demonstrated good face and content validity, and moderate to good test-retest reliability over time. From the results of the post-questionnaire interviews, only one question (Q19) required minor change in the wordings used, where participants expressed that the word "die" was too 'strong' and alternative wording was suggested. This finding is consistent with a previous local study (21). Otherwise, the CODE-EM was largely wellreceived by our pilot cohort and did not cause emotional distress in the vast majority despite death being considered generally taboo in the local population who have disparate 
cultural and religious beliefs $(22,23)$.

We observed good internal consistency for items under "ENVIRONMENT" (Cronbach's $\alpha=0.84$ ) and "CARE" (Cronbach's $\alpha=0.73$ ). This is especially important in ED where overcrowding with packed trolleys and lack of privacy for grieving are frequent issues $(6,24,25)$. Assessing quality of care under these 2 components will be paramount for improvement.

Asians are known to have different perspectives about death and are generally phobic of discussing death openly $(22,23)$. While the main core constituents of a "good death" such as alleviation of pain and the need for closure remains the same among different ethnicities, there are variations in degree of importance of these elements due to underlying cultural and religious diversity that shape an individual's experience (26). Additionally, the cultural diversity also means that death and grief experiences are handled differently among family members of various ethnicities (23). To ensure a "good death", fulfilment of palliative care needs of imminent dying patients in the ED is becoming more pressing due to the growing number of acutely ill ageing population (27). Moreover, barriers to implementation of end-of-life care in the ED have been well recognised $(5,6,28)$. Such challenges include a fast-paced environment with limited information at-hand, lack of rapport and relationship with patients on regular palliative care followup, the default "save-all" mentality among ED physicians and perceived difficulty in dealing with bereaved family members (28). In this pilot, we have shown that the CODE$\mathrm{EM}$ questionnaire is a valid and reliable instrument to assess quality of end-of-life care both in the Asian context and emergency setting. Knowledge on deficiencies will facilitate future infrastructure planning and enhanced care pathways. This information can be used to improve emergency endof-life care in various EDs across the globe.

In our pilot study, 6 out of the 7 participants who felt distressed actually commented the timing was appropriate. It is possible that bereaved next-of-kin may find it consoling and therapeutic to participate in such surveys to talk about their experience, which may aid in emotional healing and closure $(29,30)$. Given the sensitive nature of the topic, there is no good and appropriate time, as evident by the lack of consensus among our study cohort on when is the best time. Yet, it is also important to minimise recall bias and the assessment should be conducted as early as possible.

The default focus of ED physicians is to provide aggressive care to "reverse" death, which may inadvertently lead to futile care and may not alleviate suffering of the dying (31). Understanding the perspectives of the next-ofkin using CODE-EM on how their loved ones were cared for may encourage change in practice mentality among ED physicians. Components in CODE-EM can also allow us to identify if emergency physicians are deficient in specific domains such as pain management or communications. These results can effect targeted changes in training syllabus in the emergency residency programme, with added focus and specialised courses on areas of inadequacies.

Apart from medical management, communications including addressing emotions and spiritual needs is an important component in end-of-life care. While previous qualitative studies have shown ED personnel to be lacking in such communications $(32,33)$, our assessment tool will quantify the extent of inadequacy from the perspectives of bereaved family members. The CODE-EM questionnaire will allow us to pinpoint shortcomings in various aspects of ED palliative care, especially in terms of care, communications and infrastructure. Following this pilot, we have proceeded with a multicentre study using CODE-EM to evaluate the quality of end-of-life care provided in the ED (15). Our study findings in a multicultural Singapore will advise potential barriers and areas for improvement in palliative care among ED patients internationally.

\section{Strengths and limitations}

One of the strengths of our study include generalisability in our local population, as the ethnic distribution in our pilot mirrors the proportions of each race in Singapore (34). Also, our cohort comprised an almost equivalent proportion of male $(46.7 \%)$ and female $(53.3 \%)$ participants, which would give a good representation of acceptability and emotional effects from both genders.

In addition, as opposed to the original $\mathrm{CODE}^{\mathrm{TM}}$ validation study in which the relatives were enrolled 2 to 3 months after bereavement (9), our participants were approached at the bedside in ED while their loved ones were acutely ill. This may add to their emotional burden but would have reduced recall bias with real-time evaluation.

Our study has its limitations. First, our sample size is relatively small with 30 participants. As this was a pilot phase of a larger prospective multi-centre study (15), our main aim was to assess feasibility and validity of using this questionnaire in our population with maximum achievable sample size within our specified timeframe. The study results showed our participants were quite representative of our local population in terms of the gender and 
ethnicity distribution (34). Further, we achieved a response rate of $76.9 \%$ among eligible participants based on our selection criteria and a fairly high retest participation rate of $73.3 \%$ to assess test-retest reliability. Second, although different ethnic groups have been found to have similar ease in discussing death (21), the predominance of Chinese ethnicity in our study may have resulted in underrepresentation of other ethnic groups. Hence, the results may not be applicable in countries with dissimilar ethnic proportions.

Third, while we tried to provide more robust data by adopting two methods of statistical testing (percentage agreement and kappa) for test-retest reliability, kappa measures showed extreme or negative values in some questions and we could only rely on raw percentage agreement. Although kappa is commonly used to measure agreement and has the advantage of not being based on probabilistic model (20), it performs poorly when marginal distributions are very asymmetric and may be difficult to interpret (35). When kappa is inadequate in certain questions, we used percentage agreement to supplement such limitation.

Fourth, we only observe moderate consistency in "COMMUNICATION". This could be related to a slightly different angle of the questions and fewer items within this construct, as we had to ensure that the questionnaire was of an acceptable length in light of the emotional distress the participants could be facing. However, item-total scores for both Q15 and Q16 were more than 0.4, which indicated very good discrimination (36). This suggests that the items had high inter-item correlations and worked well together as individual scales.

\section{Conclusions}

This pilot study shows CODE-EM may be a valid and reliable evaluation tool for assessing quality of end-of-life care among Asian ED patients. It may help us understand the perspectives of the bereaved next-of-kin on the quality of end-of-life care rendered in the EDs and in a real-time fashion at patients' bedside, minimising recall bias. Our prospective multicentre study will further advise current barriers so that improvements can be made to better endof-life care for ED patients internationally.

\section{Acknowledgments}

We would like to thank our research coordinators, Ms
Eileen Tay, Ms Wei Lin Chua and Ms Lina Amirah Amir Ali for their assistance in the conduct of this study, and our colleagues at the emergency departments of National University Hospital, Changi General Hospital and Khoo Teck Puat Hospital for their support.

Funding: This study was funded by the National Medical Research Council's Health Services Research Grant, from Ministry of Health, Singapore. The grant was awarded to Dr. Rakhee Yash Pal (grant number: HSRG-EoL17Jun001).

\section{Footnote}

Reporting Checklist: The authors have completed the STROBE reporting checklist. Available at http://dx.doi. org/10.21037/apm-21-380

Data Sharing Statement: Available at http://dx.doi. org/10.21037/apm-21-380

Peer Review File: Available at http://dx.doi.org/10.21037/ apm-21-380

Conflicts of Interest: All authors have completed the ICMJE uniform disclosure form (available at http://dx.doi. org/10.21037/apm-21-380). CRM is funded by a Yorkshire Cancer Research 'CONNECTS' Fellowship. RYP reports grants from the National Medical Research Council, Ministry of Health, Singapore. The other authors have no conflicts of interest to declare.

Etbical Statement: The authors are accountable for all aspects of the work in ensuring that questions related to the accuracy or integrity of any part of the work are appropriately investigated and resolved. The study was conducted in accordance with the Declaration of Helsinki (as revised in 2013). The study was approved by the National Healthcare Group Domain Specific Review Board (DSRB reference no: 2018/00838) and informed consent was taken from all individual participants.

Open Access Statement: This is an Open Access article distributed in accordance with the Creative Commons Attribution-NonCommercial-NoDerivs 4.0 International License (CC BY-NC-ND 4.0), which permits the noncommercial replication and distribution of the article with the strict proviso that no changes or edits are made and the original work is properly cited (including links to both the formal publication through the relevant DOI and the license). 
See: https://creativecommons.org/licenses/by-nc-nd/4.0/.

\section{References}

1. United Nations, Department of Economic and Social Affairs, Population Division. World Popul Ageing 2017 Highlights 2017.

2. Smith AK, McCarthy E, Weber E, et al. Half of older Americans seen in emergency department in last month of life; most admitted to Hospital, and many die there. Health Aff (Millwood) 2012;31:1277-85.

3. Grudzen CR, Richardson LD, Morrison M, et al. Palliative Care Needs of Seriously Ill, Older Adults Presenting to the Emergency Department. Acad Emerg Med 2010;17:1253-7.

4. European Society for Emergency Medicine. European recommendations for end-of-life care for adults in Departments of Emergency Medicine. 2017.

5. Gloss K. End of life care in emergency departments: a review of the literature. Emerg Nurse 2017;25:29-38.

6. Beckstrand RL, Wood RD, Callister LC, et al. Emergency nurses' suggestions for improving end-of-life care obstacles. J Emerg Nurs 2012;38:e7.

7. Smith AK, Fisher J, Schonberg MA, et al. Am I Doing the Right Thing? Provider Perspectives on Improving Palliative Care in the Emergency Department. Ann Emerg Med 2009;54:86-93.e1.

8. Chor WPD, Wong SYP, Ikbal MFBM, et al. Initiating End-of-Life Care at the Emergency Department: An Observational Study. Am J Hosp Palliat Care 2019;36:941-6.

9. Mayland CR, Lees C, Germain A, et al. Caring for those who die at home: The use and validation of "Care Of the Dying Evaluation" (CODE) with bereaved relatives. BMJ Support Palliat Care 2014;4:167-74.

10. Barnato AE, Anthony DL, Skinner J, et al. Racial and Ethnic Differences in Preferences for End-of-Life Treatment. J Gen Intern Med 2009;24:695-701.

11. Johnson KS, Kuchibhatla M, Tulsky JA. What Explains Racial Differences in the Use of Advance Directives and Attitudes Toward Hospice Care? J Am Geriatr Soc 2008;56:1953-8.

12. Phua J, Kee ACL, Tan A, et al. End-of-life care in the general wards of a Singaporean hospital: An Asian perspective. J Palliat Med 2011;14:1296-301.

13. Dutta O, Lall P, Patinadan PV, et al. Patient autonomy and participation in end-of-life decision-making: An interpretive-systemic focus group study on perspectives of Asian healthcare professionals. Palliat Support Care 2020;18:425-430.

14. Lamba S, Mosenthal AC. Hospice and Palliative Medicine: A Novel Subspecialty of Emergency Medicine. J Emerg Med 2012;43:849-53.

15. Yash Pal R, Kuan WS, Tiah L, et al. End-of-life management protocol offered within emergency room (EMPOWER): study protocol for a multicentre study. BMJ Open 2020;10:e36598.

16. Poon CH. Public healthcare sector to be reorganised into 3 integrated clusters, new polyclinic group to be formed. The Straits Times 2017. Available online: https://www. straitstimes.com/singapore/health/public-healthcaresector-to-be-reorganised-into-3-integrated-clusters-new

17. Karnofsky D, Burchenal J. The clinical evaluation of chemotherapeutic agents in cancer. Eval Chemother Agents 1949;191-205.

18. Lee J. Conducting cognitive interviews in cross-national settings. Assessment 2014;21:227-40.

19. Landis JR, Koch GG. The measurement of observer agreement for categorical data. Biometrics 1977;33:159.

20. Viera AJ, Garrett JM. Understanding interobserver agreement: the kappa statistic. Fam Med 2005;37:360-3 .

21. Wee HL, Li SC, Xie F, et al. Are Asians comfortable with discussing death in health valuation studies? A study in multi-ethnic Singapore. Health Qual Life Outcomes 2006;4:93.

22. Braun KL. Death and dying in four Asian American cultures: a descriptive study. Death Stud 1997;21:327-59.

23. Yick AG, Gupta R. Chinese cultural dimensions of death, dying, and bereavement: focus group findings. J Cult Divers 2002;9:32.

24. Trzeciak S. Emergency department overcrowding in the United States: an emerging threat to patient safety and public health. Emerg Med J 2003;20:402-5.

25. Beckstrand RL, Rasmussen RJ, Luthy KE, et al. Emergency nurses' perception of department design as an obstacle to providing end-of-life care. J Emerg Nurs 2012;38:e27-e32.

26. Krikorian A, Maldonado C, Pastrana T. Patient's Perspectives on the Notion of a Good Death: A Systematic Review of the Literature. J Pain Symptom Manage 2020;59:152-64.

27. Emanuel EJ, Emanuel LL. The promise of a good death. Lancet 1998;351:SII21-SII29.

28. Chan GK. End-of-life models and emergency department care. Acad Emerg Med 2004;11:79-86.

29. Koffman J, Higginson IJ, Hall S, et al. Bereaved relatives' 
views about participating in cancer research. Palliat Med 2012;26:379-83.

30. Germain A, Mayland CR, Jack BA. The potential therapeutic value for bereaved relatives participating in research: An exploratory study. Palliat Support Care 2016;14:479-87.

31. Marck CH, Weil J, Lane H, et al. Care of the dying cancer patient in the emergency department: findings from a National survey of Australian emergency department clinicians. Intern Med J 2014;44:362-8.

32. Zhang Y, Yash Pal R, Tam WSW, et al. Spiritual perspectives of emergency medicine doctors and nurses in caring for end-of-life patients: A mixed-method study. Int

Cite this article as: Chua MT, Kuan WS, Zheng CQ, Tiah L, Kumar R, Wong YKY, Lin J, Liang S, Mayland CR, Shi L, Ibrahim I, Yash Pal R. Validation of "Care of the Dying Evaluation" in Emergency Medicine (CODE-EM): pilot phase of end-of-life management protocol offered within emergency room (EMPOWER) study. Ann Palliat Med 2021;10(6):61456155. doi: 10.21037/apm-21-380
Emerg Nurs 2018;37:13-22.

33. McBrien B. Nurses' provision of spiritual care in the emergency Setting - An Irish Perspective. Int Emerg Nurs 2010;18:119-26.

34. Population in brief. Strateg Group, Singapore. Available online: https://www.strategygroup.gov.sg/media-centre/ publications/population-in-brief

35. Delgado R, Tibau XA. Why Cohen's Kappa should be avoided as performance measure in classification. PLoS One 2019;14:e0222916.

36. Pope G. Psychometrics 101: Item Total Correlation. Available online: https://www.questionmark.com/itemtotal-correlation/ 


\section{Appendix 1 CODE-EM Family Survey Tool}

CODE-EM Questionnaire

1 There was enough help with nursing care in the ED, such as giving medicines, changing diapers and helping the patient find a comfortable position in bed.

2 The bed area in the ED and surrounding environment was comfortable for the patient.

3 The bed area in the ED and surrounding environment was comfortable for the family.

The bed area in the ED and surrounding environment had adequate privacy for the patient

Did you have confidence and trust in the ED nurses who were caring for the patient?

Did you have confidence and trust in the ED doctors who were caring for the patient?

In your opinion, during the patient's stay in the ED, did the patient appear to be in pain?

$8 \quad$ In your view, did the doctors and nurses in the ED do enough to help relieve the pain?

In your opinion, during the stay in the ED, did the patient appear to be restless?

In your view, did the doctors and nurses in the emergency department do enough to help relieve the restlessness?

11 In your opinion, during the stay in the ED, did the patient appear to have a "noisy rattle when breathing?

12 In your view, did the doctors and nurses in the ED do enough to help relieve the "noisy rattle" when breathing?

13 In your opinion, during the stay in the emergency department, did the patient appear to have difficulty breathing?

14 In your view, did the doctors and nurses in the emergency department do enough to help relieve the breathing difficulty?

15 During the time in the ED, the patient's care and treatment was discussed with you and/or the family.

16 The healthcare team at the ED explained the patient's condition and treatment in a way you found easy or difficult to understand.

17 How would you assess the overall level of emotional support given to you by the ED healthcare team?

18 The ED healthcare team discussed the patient's religious or spiritual needs.

19 Were you told that the patient would be likely to die soon?

19a) If yes, who told you s/he was likely to pass away soon?

20 Did a member of the ED healthcare team talk to you about what to expect during the dying process (e.g. what symptoms may arise)?

20a) If yes $\rightarrow$ Was the discussion about what to expect during the dying process helpful?

20b) If no $\rightarrow$ Would a discussion about what to expect during the dying process have been helpful?

21 In your view, the patient was treated with respect and dignity by the ED doctors.

22 In your view, the patient treated with respect and dignity by the ED nurses.

23 Overall, in your opinion, were you adequately supported during the patient's stay in ED?

\begin{tabular}{|c|c|c|c|c|}
\hline $\begin{array}{c}\text { Strongly } \\
\text { agree }\end{array}$ & Agree & Neutral & Disagree & $\begin{array}{l}\text { Strongly } \\
\text { disagree }\end{array}$ \\
\hline $\begin{array}{c}\text { Strongly } \\
\text { agree }\end{array}$ & Agree & Neutral & Disagree & $\begin{array}{l}\text { Strongly } \\
\text { disagree }\end{array}$ \\
\hline $\begin{array}{c}\text { Strongly } \\
\text { agree }\end{array}$ & Agree & Neutral & Disagree & $\begin{array}{l}\text { Strongly } \\
\text { disagree }\end{array}$ \\
\hline $\begin{array}{c}\text { Strongly } \\
\text { agree }\end{array}$ & Agree & Neutral & Disagree & $\begin{array}{l}\text { Strongly } \\
\text { disagree }\end{array}$ \\
\hline
\end{tabular}

Yes, in all of them Yes, in some of them

Yes, in some of them

Yes, all of the Yes, some of the time time

No, not in any of the nurses

No, not in any of the doctors

No, s/he did not appear to be in pain

Yes, all of the time

Yes, all of the time

Yes, all of the time

Yes, all of the time

Yes, all of the time

Yes, all of the time

Yes, some of the time

Yes, some of the time

Yes, some of the time

Yes, some of the time

Yes, some of the time

Yes, some of the time

Yes, all of the time

Yes, some of the time

No, not at all Not applicable, s/he was not in pain

No, s/he did not appear to be restless

No, Not applicable, s/he not at all was not restless

No, s/he did not have a noisy rattle to the breathing

No, Not applicable, s/he not at all did not have a noisy rattle to the breathing

No, s/he did not have difficulty breathing

No, Not applicable, s/he not at all did not have difficulty breathing

\begin{tabular}{|c|c|c|c|c|}
\hline $\begin{array}{c}\text { Strongly } \\
\text { agree }\end{array}$ & Agree & Neutral & Disagree & $\begin{array}{l}\text { Strongly } \\
\text { disagree }\end{array}$ \\
\hline Very easy & Easy & Neutral & Difficult & Very Difficult \\
\hline Very poor & Poor & Fair & Good & Excellent \\
\hline $\begin{array}{l}\text { Strongly } \\
\text { agree }\end{array}$ & Agree & Neutral & Disagree & $\begin{array}{l}\text { Strongly } \\
\text { disagree }\end{array}$ \\
\hline
\end{tabular}

Yes

No

Yes

No

Yes

No

Yes

No

$\begin{array}{cllll}\begin{array}{c}\text { Strongly } \\ \text { agree }\end{array} & \text { Agree } & \text { Neutral } & \text { Disagree } & \begin{array}{l}\text { Strongly } \\ \text { disagree }\end{array} \\ \begin{array}{c}\text { Strongly } \\ \text { agree }\end{array} & \text { Agree } & \text { Neutral } & \text { Disagree } & \begin{array}{l}\text { Strongly } \\ \text { disagree }\end{array}\end{array}$

Yes

CODE-EM, Care of the Dying Evaluation - Emergency Medicine; ED, emergency department. 
Table SI Deriation of CODE-EM from original CODE questionaire
Orignal COE

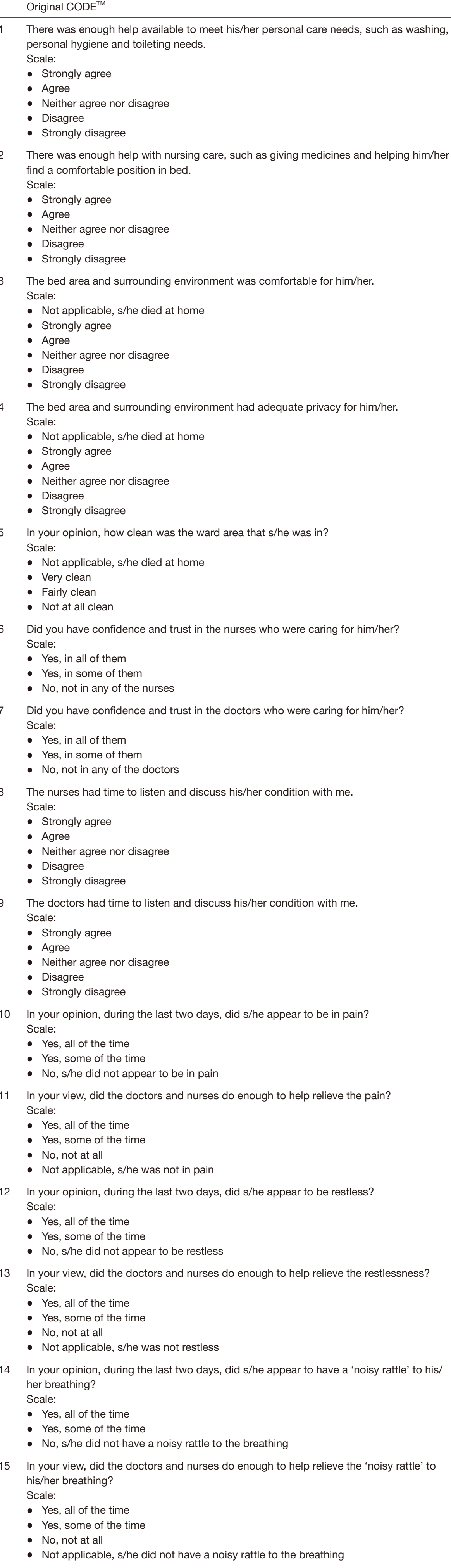

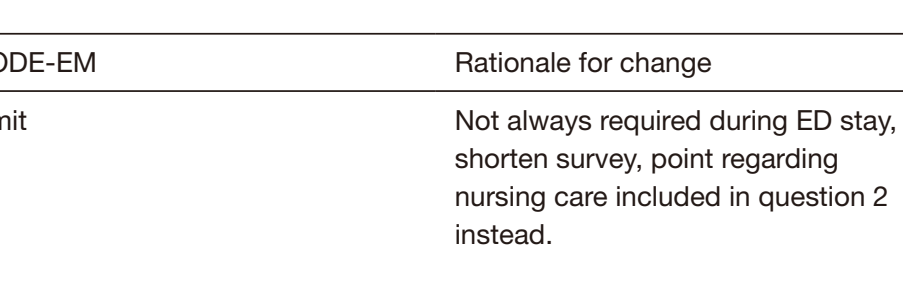

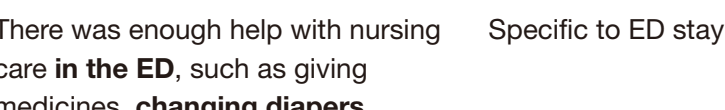

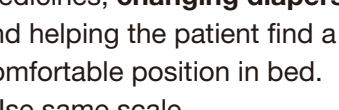

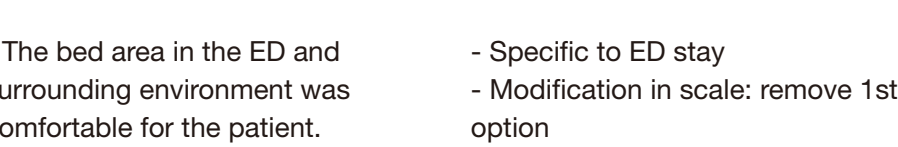

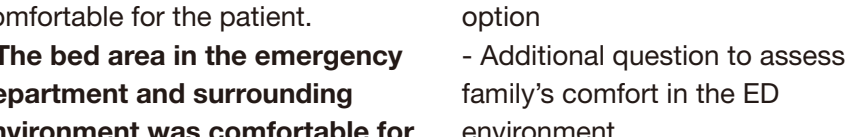
enviromme
the tanily.

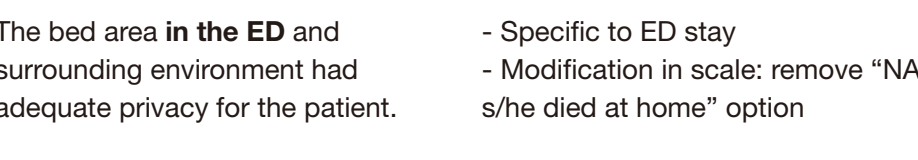

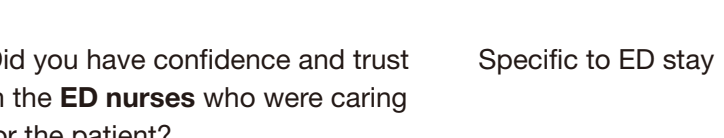
Oor the patient?
Usse same scale

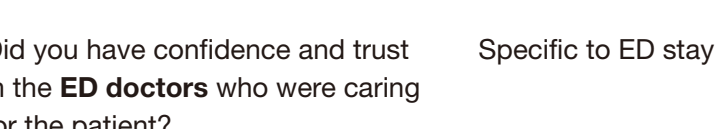
lorthe patient?
Uses same scale

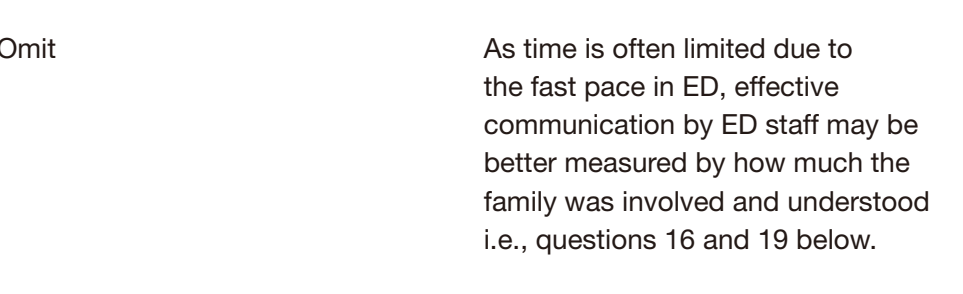

omit As above

In your ropinion, during the patients Specific to ED Stay
stay in the ED, did the patient

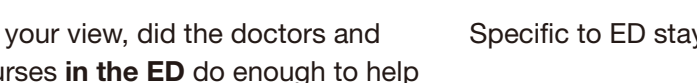
Ielive the pain?
Use same scale

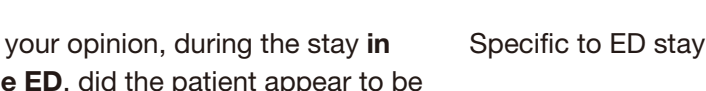

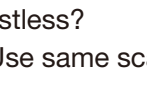

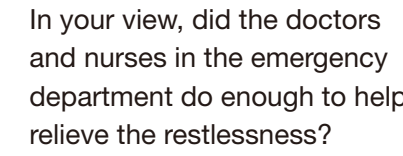

In vour opinion, during the stay

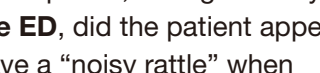

same scale

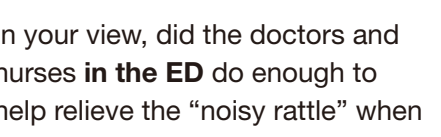

Leathin?
Jus sane scale

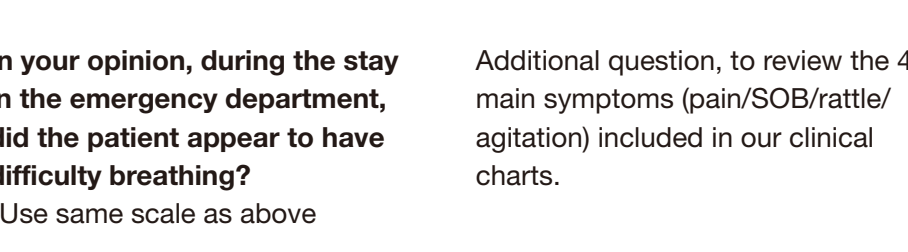

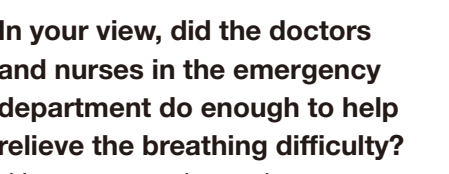

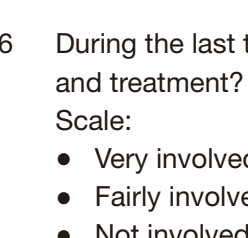

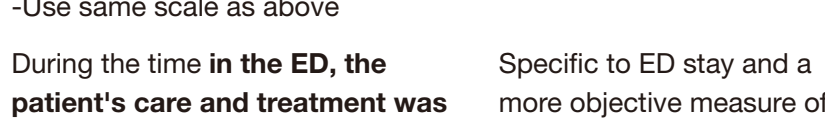

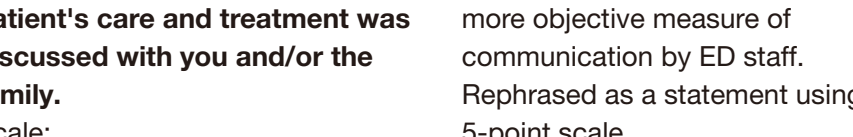

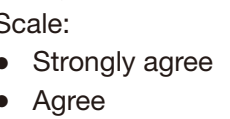

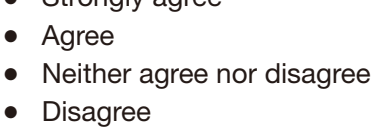

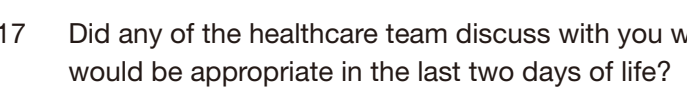
: Disarger Strongly disagree

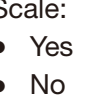

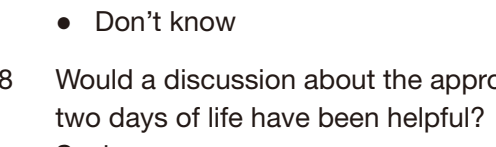

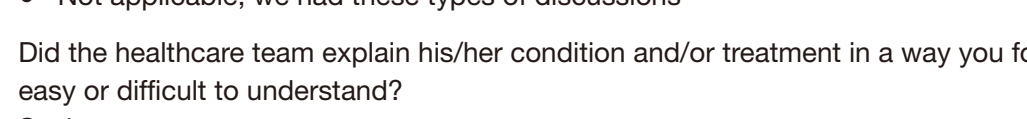

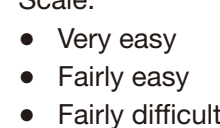

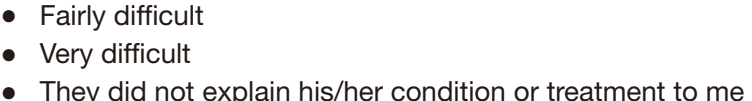

healthc
scale:
$:$ Poor
Fair
Fair
Good

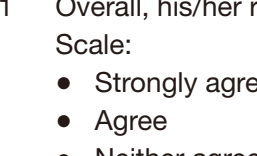

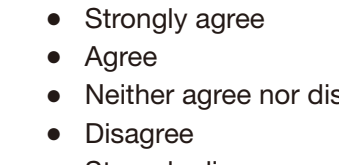

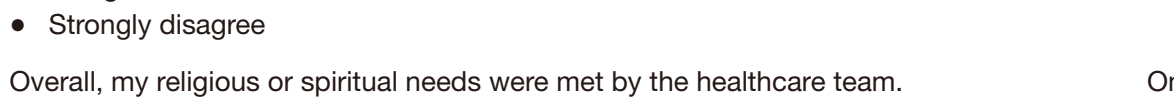

: Strongly arear

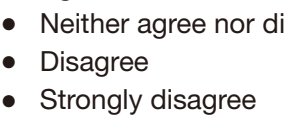

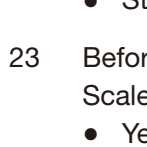

you tod she was intey to did soor

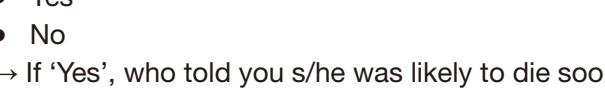

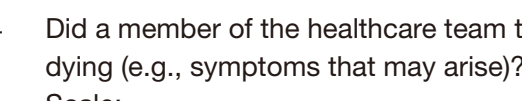

dying
Scale:
$:$ Yes
N No

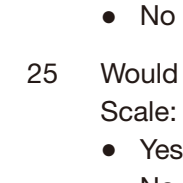

- Not appicable, we had these types of discusssors

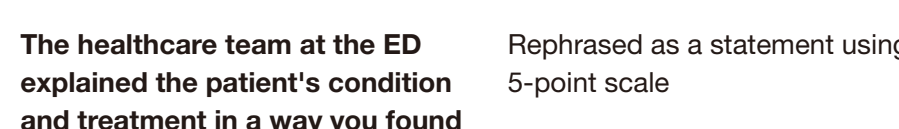

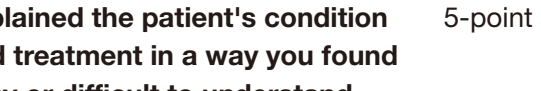
(a)

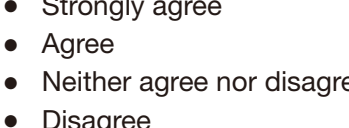

: Disagrae age nor rasagree

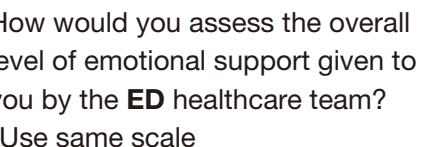

Succificto E E stay

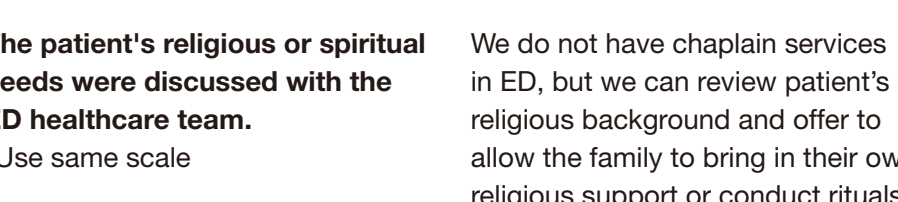

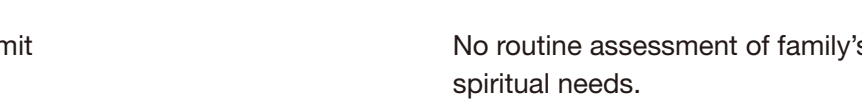

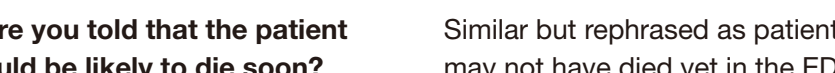

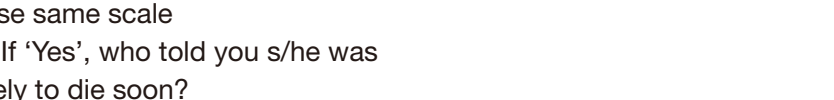

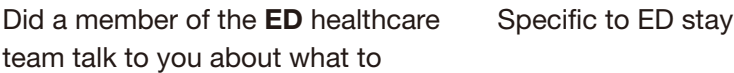

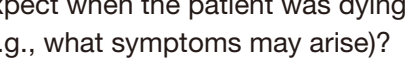

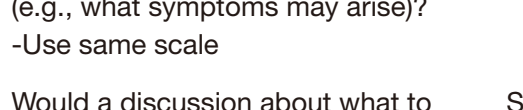

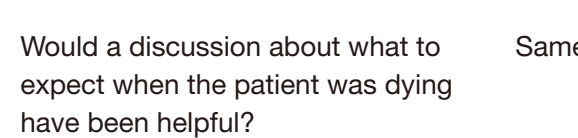

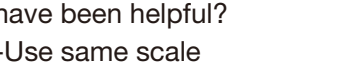

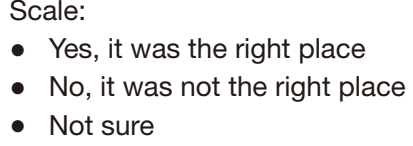

27 i was givin

:

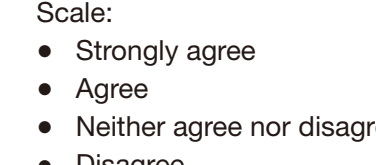

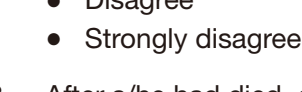

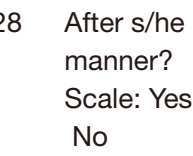

(1)

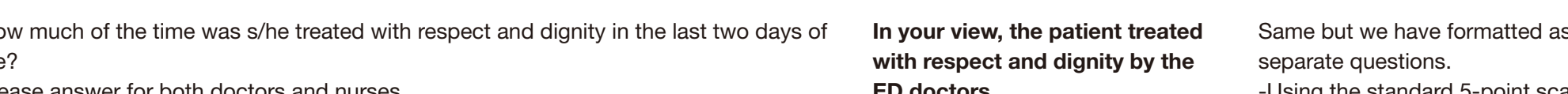

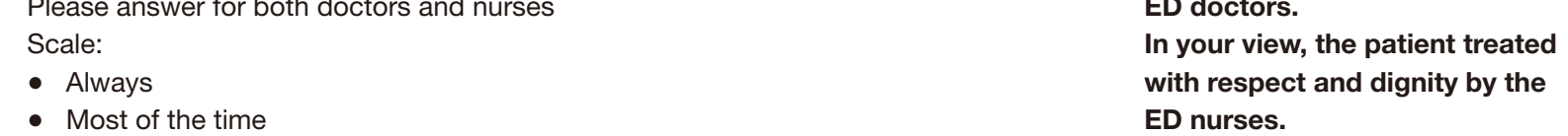

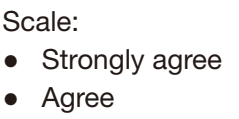

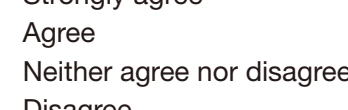
: Strongly disagree

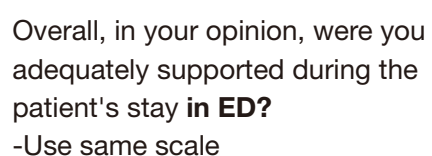

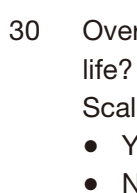




\begin{tabular}{lcccc}
\hline Questions & Yes & No & Open question results & $\begin{array}{c}\text { Actions on the } \\
\text { questionnaire revision }\end{array}$ \\
\hline $\begin{array}{l}\text { 1. Were the questions easy to understand/wording } \\
\text { was clear? }\end{array}$ & $30(100 \%)$ & $0(0 \%)$ & No actions
\end{tabular}

1a. If no, which questions?

1b. How can this be improved?

2. Did the questions make you feel emotionally distressed?

2a. If yes, which questions?

2b. How can this be improved?

3. Were any of the questions irrelevant?

3a. If yes, which questions?

3b. How can this be improved?

4. What are your thoughts on the length of this survey?

4a. If too long, which questions should be take out?

5. Was the survey conducted at an appropriate timing?

5a. If you feel the timing was inappropriate, what would be a better timing? (Other options e. should be done later in the ED/after the patient's demise/a week later, etc)

6. Any other feedback you would like to share with
$7(23.3 \%) \quad 23(76.7 \%)$

Revise the wording in

Q13, Q19

Q13: One participant noticed that his mother was struggling to breathe when he arrived at the ED. He also noticed that the space in ED was constrained, but felt it was understandable. Hence, he thought that these could be improved. (Subject ID NPP002, male)

Q19: One participant felt that the word 'die' carries a strong meaning He suggested to change to something more neutral (i.e., worsen). (Subject ID NPP007, male)

General comments:

Two participants commented that the questionnaire was generally difficult to answer as the topic was on death at a point in time when their loved ones were actively dying. Overall, the questions made them feel emotionally distressed.

"No particular question, emotionally distressed in general in view of current situation" (Subject ID KPP013, male)

"(Emotionally distressed) in general, as this concerns life and death" (Subject ID NPP011, male)

$7(23.3 \%) \quad 23(76.7 \%)$

Q5, Q6, Q7, Q18, Q19, Q20

Q5 \& Q6: One participant commented that she came to the hospital with confidence in staff/professionals and thus, should not be asked these questions. (Subject ID CPPO01, female)

Q7: One respondent felt that he was unable to tell if his grandfather was in pain as he (patient) was unconscious. He thinks that if there were no symptoms, the family would not have sent the patient to the ED, thus he felt that we could tailor the questions accordingly. (Subject ID NPP004, male)

Q18 (Subject KPP004 chose "Neutral"): "Doctor did not raise this question at all" (Subject ID KPP004, male)

Q18 (Subject KPP006 did not choose any answers): One participan felt that this question was irrelevant and may also be insensitive. (Subject ID KPP006, female)

Q19 \& Q20: One respondent mentioned that these two questions seemed to be overlapping. (Subject ID KPP001, male)

Q20: Participant felt that this question was irrelevant as he was already aware of dying process beforehand. (Subject ID KPPO03, male)

Just nice: 27 Too long: 3

$(90.0 \%)$

- "Not sure, slightly too long." (Subject ID KPP007, female)

$20(66.7 \%) \quad 10(33.3 \%)$

- "Probably best later" (Subject ID CPP002, male)

- "At least 1 day after demise" (Subject ID KPPO03, male)

- "1-2 days after admission to ward" (Subject ID KPP006, female)

- "Unsure" (Subject ID KPP008, female)

- "The timing) would depend on the condition of the patient." (Subject ID NPP003, female)

- One respondent felt that the stay in ED was not long enough and a more appropriate time would be after the patient had "completed" the stay (i.e. admitted to inpatient ward). (Subject ID NPPO04, male)

- "There is no 'good' time. It all depends on individuals' coping." (Subject ID NPP011, male)

- "A week later would be more appropriate." (Subject ID NPP012, male)

- One participant suggested that there should have been a place for the body other than the mortuary while waiting for undertaker. (Subject ID CPP001, female)

- "Range of choices should be shortened." (Subject ID KPP001, male)

- For Question 19, one respondent commented that "Yes or No" does not answer the question as the attending doctor did not explicitly state imminent demise but merely explained in terms of higher probability of death. (Subject ID KPP004, male)

- "Might be too long for others in this situation" (Subject ID KPP008, female)

- "The timing of conducting the survey can be quire subjective. But for family, it was appropriate. Waiting time for bed is too long." (Subject ID NPP001, female)

- "Length of survey might be subjective; depends on the state of mind of the interviewee. Waiting time for bed is unbearable." (Subject ID NPPO03, female)

- One participant felt that the survey should be conducted at a time when it's "not too early or too late". (Subject ID NPP005, female) 